\title{
Preface
}

\section{Guohui Lin}

Published online: 17 February 2013

(C) Springer Science+Business Media New York 2013

This special issue of the Journal of Combinatorial Optimization contains nine selected papers that were presented at the Sixth Annual International Conference on Combinatorial Optimization and Applications (COCOA 2012), held in Banff, Alberta, Canada, during August 5-9, 2012. COCOA 2012 received 57 high-quality submissions for full consideration and 33 of them were accepted for podium presentations. Among these 33 accepted papers, 17 were invited to submit their full versions to two special issues. After a substantial reviewing process we accepted eight of them to be included in this special issue, two in computational biology and the other six in graph optimization.

Zhang, Jiang and Zhu studied the parameterized complexity of the radiation hybrid map construction problem, and presented a linear kernel and an FPT algorithm; Liang and Zhang presented efficient algorithms for computing the local forest similarity between two ordered labelled forests, which can be used to locate the structurally similar regions in RNA secondary structures.

The approximability of the minimum diameter cost-constrained Steiner tree problem was studied by Ding and Xue, where a weakly FPTAS is presented for a variant in which the tree topology is part of the input; Cheng, Qiu and Shen extended the notion of surface area from vertex-centered to edge-centered in an interconnection network, and derived its explicit expression for the arrangement graph; Liu and Zhang studied the generalized multiway cut problem in trees and showed that the problem is FPT; Certain geometric properties of the Yao-Yao graphs were studied by Kanj and Xia, one of which answers partially negatively an open problem about the spanner properties of Yao-Yao graphs; Eto, Guo and Miyano generalized the notion of independent set to distance- $d$ independent set, D $d \mathrm{IS}$, and achieved several hardness and algorithmic results; Anthony and Chung examined the online bottleneck matching problem in

G. Lin $(\bowtie)$

Department of Computing Science, University of Alberta, Edmonton, AB T6G 2E8, Canada e-mail: guohui@ualberta.ca 
certain metric spaces, and used resource augmentation analysis to characterize the competitive ratios of three algorithms: greedy, permutation, and balance.

I would like to thank the authors for submitting and revising their work, and PC members and additional referees for their careful reviewing. I would also like to thank Dr. Du, the editor-in-chief, for his support of COCOA 2012. 